\title{
HGF induces the serine-phosphorylation and cell surface translocation of ROMK (Kir 1.1) channels in rat kidney cells
}

\author{
TAKASHI KATO ${ }^{1,2}$, KIYOMASA OKA ${ }^{3}$ and TOSHIKAZU NAKAMURA ${ }^{3}$ \\ ${ }^{1}$ Department of Pharmacology, Faculty of Medicine, Kindai University, Osakasayama, Osaka 589-8511; \\ ${ }^{2}$ Division of Molecular Regenerative Medicine, Department of Biochemistry, Osaka University Graduate School of \\ Medicine, Suita, Osaka 565-0871; ${ }^{3}$ Research and Development, Neurogen, Inc., Ibaraki, Osaka 567-0085, Japan
}

Received March 1, 2016; Accepted February 9, 2017

DOI: $10.3892 / \mathrm{mmr} .2017 .7969$

\begin{abstract}
Extracellular potassium homeostasis is dependent on the activity of potassium channels, which are expressed on the apical membrane of epithelial tubular cells. The renal outer medullary potassium channel (ROMK) is considered to be the major route for potassium transport into the tubule lumen. Hepatocyte growth factor (HGF) exerts multiple biological activities and is important for maintaining renal homeostasis. It is also anti-apoptotic and mitogenic for protection and recovery from ARF. Whether HGF regulates the ion channel activities remains to be elucidated, therefore, the present study aimed to investigate the modulation of HGF on the expression of ROMK in cultured renal tubular cells. NRK-52E cells were treated with recombinant HGF, however, no alterations in the total expression of ROMK were observed by western blot analysis. In examining the serine 44 phosphorylation of ROMK in NRK-52E cells, the present study observed that HGF enhanced the serine 44 phosphorylation of ROMK. In addition, to investigate whether HGF-Met signaling induces the movement of ROMK to the cell surface in NRK-52E cells, the protein constituents of cells were separated into plasma membrane and cytoplasm. Using immunofluorescence assay, the expression of ROMK on the plasma membrane was increased in the HGF-treated NRK-52E cells, which suggested that ROMK was translocated to the plasma membrane following the HGF-induced phosphorylation of serine 44. Therefore, HGF may be important in potassium excretion and perform antihyperkalemic effects through the translocation of potassium channels.
\end{abstract}

Correspondence to: Dr Takashi Kato, Department of Pharmacology, Faculty of Medicine, Kindai University, 377-2 Ohno-Higashi, Osakasayama, Osaka 589-8511, Japan

E-mail: takashi0920k@gmail.com

Key words: renal outer medullary potassium channel, hepatocyte growth factor, kidney

\section{Introduction}

Extracellular potassium homeostasis is maintained by the regulation of renal potassium excretion, and is dependent on the activity of potassium channels, which are expressed on the apical membrane of epithelial tubular cells. Encoded by the renal outer medullary potassium channel (ROMK) gene (also termed Kir 1.1 and KCNJ1) $(1,2)$, ROMK channels are considered to be the major route for the transport of potassium into the tubule lumen $(3,4)$. As ROMK channels usually exhibit a high probability of being open, the physiologic augmentation of channel activity is controlled by hormones and dietary potassium, and is achieved predominantly by regulated alterations in the number of active channels on the plasma membrane (5).

Hepatocyte growth factor (HGF) exerts multiple biological activities, including mitogenic, motogenic and morphogenic activities, on a variety of cells through its receptor, Met. The multiple functions of HGF are important in organ regeneration (6). In particular, HGF is important for maintaining renal homeostasis $(7,8)$. The levels of HGF in the plasma increase following renal ischemia, and the inhibition of HGF-Met signaling enhances tubular apoptosis in the early stage of acute renal failure (ARF) (7) and inhibits the proliferation of tubular cells in the late stage (8). However, whether HGF regulates the ion channel activities remains to be elucidated, therefore, the present study investigated the modulation of HGF on the expression, phosphorylation and translocation of ROMK in renal tubular cells.

\section{Materials and methods}

Materials. Human recombinant $\mathrm{HGF}$ was purified from the medium of Chinese hamster ovary (CHO) cells transfected with human HGF cDNA of the 5-amino acid deleted type ( $>98 \%$ of purity on SDS-PAGE) as previously described $(7,8)$. The following antibodies were used: Anti-Tyr1234/1235-phosphorylated Met (D26; cat. no. 3126; Cell Signaling Technology, Inc., Beverly, MA, USA), anti-Met (SP260; cat. no. sc-162; Santa Cruz Biotechnology, Inc., Danvers, MA, USA), anti-Met (B2; cat. no. sc-8057; Santa Cruz Biotechnology, Inc.), anti-ROMK (cat. no. APC001; Alomone Labs, Ltd., Jerusalem, Israel), anti-pS44/25-ROMK (cat. no A1121; Assay Biotech Co., Inc., Sunnyvale, CA, USA), 
anti-E-cadherin (cat.no. C20820,BD Biosciences, San Jose, CA, USA), anti-GAPDH (cat. no. sc-32233, SCB), and anti- $\beta$-actin (cat. no. A1978; Sigma-Aldrich; Merck KGaA, Darmstadt, Germany). Membrane-bound ROMK and E-cadherin were biotinylated with Sulfo-NHS-LC-biotin (Thermo Fisher Scientific, Inc., Waltham, MA, USA) according to the manufacturer's protocol. The NRK-52E normal rat kidney epithelial cell line was obtained from American Type Culture Collection (Manassas, VA, USA) cultured in Dulbecco's modified Eagle's medium (DMEM; Nacalai Tesque, Inc., Kyoto, Japan) supplemented with $10 \%$ fetal calf serum. Cells were maintained and grown at $37^{\circ} \mathrm{C}$ in $5 \% \mathrm{CO}_{2}$ according to the supplier's recommendations.

Immunoblotting. The cells were lysed on ice with lysis buffer, containing $50 \mathrm{mM}$ Tris- $\mathrm{HCl}(\mathrm{pH} 7.5), 150 \mathrm{mM} \mathrm{NaCl}$, $1 \%$ Triton X-100, $5 \mathrm{mM}$ EDTA, protease inhibitor cocktail, $2 \mathrm{mM} \mathrm{Na}_{3} \mathrm{VO}_{4}$ and $50 \mathrm{mM} \mathrm{NaF}$. The protein concentrations of cell lysates were measured by BCA protein assay (Thermo Fisher Scientific, Inc.). For immunoblot assay of Met and Tyr 1234/1235-phosphorylated Met, immunoprecipitation was performed as previously reported $(8,9)$. The cell lysates (300 $\mu \mathrm{g} / \mathrm{sample})$ were incubated with anti-Met antibody (B2) at $4^{\circ} \mathrm{C}$ overnight and then precipitated with protein G/A (GE Healthcare Life Sciences, Chalfont, UK) for $2 \mathrm{~h}$ at $4^{\circ} \mathrm{C}$. The immunoprecipitated lysates were then subjected to SDS-PAGE and transferred onto PVDF membranes. The primary antibodies (Met and Tyr 1234/1235-phosphorylated Met) were applied to PVDF membranes overnight at $4^{\circ} \mathrm{C}$ at a concentration of 1:4,000, followed by a second reaction with horseradish peroxidase-labeled antibodies (Dako, Glostrup, Denmark) at a concentration of 1:4,000 for $1 \mathrm{~h}$ at room temperature. For the immunoblot assay of ROMK, serine 44 phosphorylated ROMK and E-cadherin, the cell lysates were subjected to SDS-PAGE, and then detected as immunoblots in a similar method to total and tyrosine 1234/1235-phosphorylated Met. (1:4,000). The $\beta$-actin and GAPDH antibodies were applied overnight at $4^{\circ} \mathrm{C}$ at a concentration of 1:8,000, and followed by a second reaction horseradish peroxidase-conjugated antibodies (Dako) at a concentration of 1:8,000 for $1 \mathrm{~h}$ at room temperature. The signals were visualized on PVDF membranes, using a kit (ECL system; GE Healthcare Life Sciences). Densitometric quantification of the scanned band intensities was performed by digitizing each band via densitometry using ImageJ software version $1.47 \mathrm{t}$ (National Institutes of Health, Bethesda, MD, USA) on a Macintosh computer.

Immunofluorescence. The cells on cover glass were fixed with $4 \%$ paraformaldehyde in PBS for $15 \mathrm{~min}$, permeabilized with $0.2 \%$ Triton X-100 in PBS for 5 min, and blocked with $5 \%$ goat serum in PBS for $1 \mathrm{~h}$ at room temperature. The cells were then incubated with primary antibodies (ROMK and serine 44 phophorylated ROMK) overnight at $4{ }^{\circ} \mathrm{C}$ at a concentration of $1: 200$, followed by incubation with secondary antibodies conjugated to Alexa Fluor 488 (Thermo Fisher Scientific, Inc.) at room temperature for $20 \mathrm{~min}$ at a concentration of 1:600. The nuclei were stained with propidium iodide (PI; Sigma-Aldrich; Merck KGaA). Following washing with PBS, the cells were mounted with crystal mount (Biomeda Corporation, Foster City, CA, USA), and observed under an LSM5 Pascal confocal microscope (Zeiss AG, Oberkochen, Germany).

Statistical analysis. Student's t-test was performed to evaluate the significant differences of each group using Microsoft Excel for Mac 2011. Data are expressed as mean \pm standard deviation. $\mathrm{P}<0.05$ was considered to indicate statistically significant.

\section{Results}

HGF does not alter the expression of ROMK, but phosphorylates serine-44 of ROMK in rat kidney epithelial cells. HGF led to an increase of Tyr 1234/1235-phosphorylated Met in the NRK-52E cells, determined using western blot analysis (Fig. 1), confirming it had an effect on the NRK-52E cells. Whether HGF modulated the expression of ROMK was then investigated. Following the addition of NRK-52E cells to $10 \mathrm{ng} / \mathrm{ml}$ of recombinant $\mathrm{HGF}$, the expression of ROMK was measured using western blot analysis. However, no alterations in the total expression of ROMK were observed (Fig. 2A). ROMK activity is dependent on direct phosphorylation by protein kinase A (PKA). PKA-acceptor sites in ROMK, embedded within the cytoplasmic $\mathrm{NH}_{2}-(\mathrm{Ser}-44)$ and $\mathrm{COOH}$-termini (Ser-219 and Ser-313) require phosphorylation for full channel activity (10). The phosphorylation of the two $\mathrm{COOH}$-terminal sites is required to maintain a high probability of the channel being in an open state (10). The phosphorylation of the $\mathrm{NH}_{2}$-termination site has no effect on the probability of channel opening, however, it appears to control the number of active channels on the cell surface (10). In the present study, serine 44 phosphorylation of ROMK in the NRK-52E cells was examined. Treatment with HGF enhanced the level of serine 44 phosphorylated ROMK (Fig. 2B). The results of the immunofluorescence assay also demonstrated the upregulated serine 44 phosphorylation of ROMK (Fig. 2C).

HGF translocates ROMK into the plasma membrane in normal kidney cells. To investigate whether HGF-Met signaling induced the movement of ROMK to the cell surface in NRK-52E cells, the present study separated the protein constituents of cells into plasma membrane and cytoplasm proteins. The present study confirmed that the proteins derived from plasma membrane and cytoplasm were E-cadherin and GAPDH using an immunoblot assay (Fig. 3A). The results of the western blot analysis, revealed that the expression of ROMK on the plasma membrane was increased in the HGF-treated NRK-52E cells (Fig. 3A). In addition, the results of the immunofluorescence analysis indicated that HGF induced the translocation of ROMK into the plasma membrane (Fig. 3B).

\section{Discussion}

By measuring the cell surface density and functional activity of wild-type and phosphorylation-site mutant ROMK channels, Yoo et al (11) suggested that the phosphorylation of serine 44 markedly increases functional channel density by recruiting on the plasma membrane, rather than by activating silent channels already present on the plasma membrane. In the present study, HGF enhanced ROMK localization on the plasma membrane through the phosphorylation of serine 44 . 


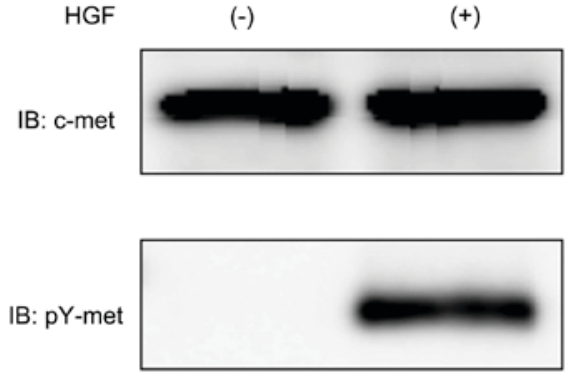

Figure 1. HGF activates Met in normal kidney cells. Western blot analysis was used with anti-phosphorylated tyrosine or anti-c-Met antibodies in NRK52E cells. Total c-Met was used as a loading control. IB, immunoblot; HGF, hepatocyte growth factor.

Close inspection of the $\mathrm{NH}_{2}$-terminal PKA site in ROMK has revealed that it also falls within a canonical serum- and glucocorticoid-regulated kinase (SGK1) phosphorylation sequence (11), which suggests that the channels, and serine 44 in particular, may also be a target of SGK1. SGK1 is a member of the protein kinase B/Akt family of serine/threonine kinases in the renal collecting duct $(12,13)$, which has been shown to regulate the cell surface expression of epithelial $\mathrm{Na}^{+}$channels. Several pathways regulate SGK1, including growth factor signaling, comprising HGF, and stress-mediated signaling, and this is achieved through involvement of the signaling pathways in the regulation of cell survival, and cell-cell and cell-matrix interactions (14). Therefore, HGF activated ROMK through the activation of SGK1, and may have also controlled the number of functional ROMK at the plasma membrane.

Clinical crush syndrome occurs as a consequence of traumatic events, for example during accidents. As a result of muscle compression, myocytes are damaged, and this is followed by the release of intracellular constituents into the systemic circulation. Rhabdomyolysis, due to substantial cell necrosis in injured muscles, causes the release of intracellular materials, including potassium and myoglobin, into the systemic circulation, which are important in early-and late-phase life-threatening complications, including cardiac arrhythmia, myoglobinuric renal failure and systemic inflammation (15). ARF due to crush-induced rhabdomyolysis is the second most frequent cause of mortality following traumatic impact in catastrophic earthquakes (16). The repeated administration of HGF has been found to inhibit acute tubular necrosis in the renal cortex, improve renal dysfunction and protect rats from death caused by severe ARF (17). The exogenous supply of HGF may be efficacious in combating ARF with acute tubular necrosis, particularly rhabdomyolysis-induced ARF (17). HGF has mitogenic, motogenic and morphogenic functions, which accelerate the regeneration of tubular epithelial cells and the reconstruction of normal tissue architecture of the kidney. It also stimulates cation transport through the activation of tubular $\mathrm{Na}^{+}-\mathrm{K}^{+}$-ATPase (18). HGF may also be important in potassium excretion and perform antihyperkalemic effects through translocation of the ROMK potassium channel.

In the present study, it was found that HGF treatment led to the increased serine 44 phosphorylation of ROMK and the translocation of ROMK into the nucleus in normal kidney cells. Future investigations aim to elucidate the effects of HGF on potassium excretion.
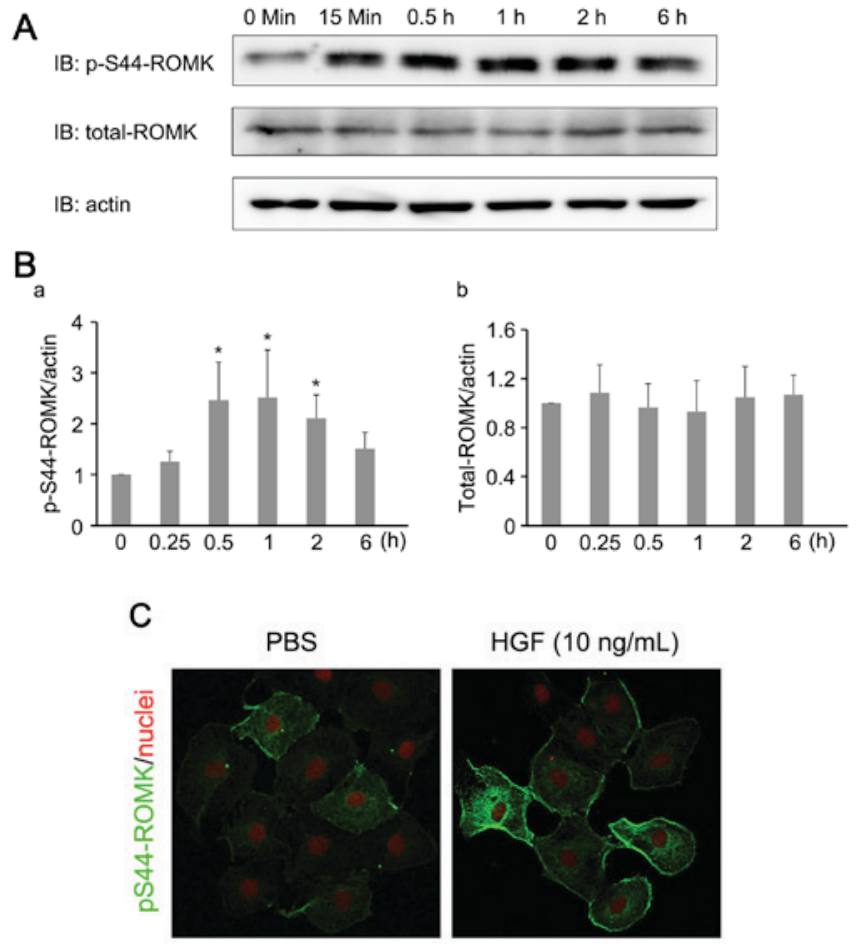

Figure 2. HGF upregulates the serine-phosphorylation of ROMK. (A) Results of western blot analysis for the serine-phosphorylation of ROMK in NRK-52E cells following treatment with $10 \mathrm{ng} / \mathrm{ml} \mathrm{HGF}$. (B) Alterations in protein expression levels of (a) serine44-phosphorylated ROMK and (b) total ROMK were detected using anti-phosphorylated-ROMK (Ser44) and anti-ROMK antibodies $(n=3)$. ${ }^{*} \mathrm{P}<0.05$ vs. 0 h. (C) Immunofluorescence images of serine-phosphorylated ROMK (green) in HGF-treated NRK-52E cells at $2 \mathrm{~h}$. Magnification, $\mathrm{x} 400$. The nuclei were counterstained with propidium iodide (red). IB, immunoblot; HGF, hepatocyte growth factor; ROMK, renal outer medullary potassium channel; p-, phosphorylated.

\section{A}

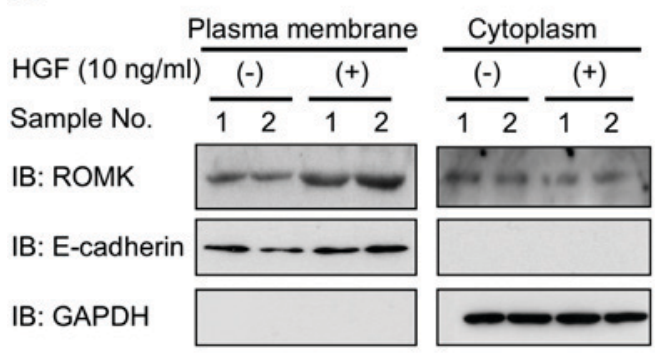

B

ROMK/nuclei

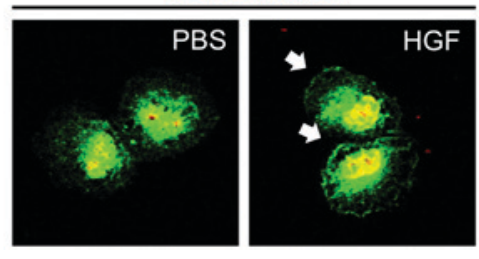

Figure 3. HGF enhances ROMK localization at the plasma membrane (A) Results of western blot analysis of ROMK localization in NRK-52E cells cultured for $1 \mathrm{~h}$ in the presence of $10 \mathrm{ng} / \mathrm{ml} \mathrm{HGF}$. Anti-E-cadherin and anti-GAPDH served as protein sample controls of the plasma membrane or cytoplasm. (B) Immunofluorescence images of ROMK (green) in HGF-treated NRK-52E cells at $2 \mathrm{~h}(\mathrm{x} 400)$. The nuclei were counterstained with propidium iodide (red). HGF, hepatocyte growth factor; ROMK, renal outer medullary potassium channel. Arrows indicate ROMK localized at plasma membrane in NRK-52E cells. HGF, hepatocyte growth factor; ROMK, renal outer medullary potassium channel. 


\section{Acknowledgements}

The present study was supported by a grant from the Ministry of Education, Culture, Sports, Science and Technology of Japan (grant no. 14454911 to Dr Takashi Kato).

\section{References}

1. Ho K, Nichols CG, Lederer WJ, Lytton J, Vassilev PM, Kanazirska MV and Hebert SC: Cloning and expression of an inwardly rectifying ATP-regulated potassium channel. Nature 362: 31-38, 1993.

2. Lu M, Wang T, Yan Q, Yang X, Dong K, Knepper MA, Wang W, Giebisch G, Shull GE and Hebert SC: Absence of small conductance $\mathrm{K}+$ channel (SK) activity in apical membranes of thick ascending limb and cortical collecting duct in ROMK (Bartter's) knockout mice. J Biol Chem 277: 37881-37887, 2002.

3. Giebisch G: Renal potassium transport: Mechanisms and regulation. Am J Physiol 274: F817-F833, 1998.

4. Palmer LG: Potassium secretion and the regulation of distal nephron K channels. Am J Physiol 277: F821-F825, 1999.

5. Wang W, Sackin H and Giebisch G: Renal potassium channels and their regulation. Annu Rev Physiol 54: 81-96, 1992.

6. Nakamura T and Mizuno S: The discovery of hepatocyte growth factor (HGF) and its significance for cell biology, life sciences and clinical medicine. Proc Jpn Acad Ser B Phys Biol Sci 86: 588-610, 2010.

7. Mizuno S and Nakamura T: Prevention of neutrophil extravasation by hepatocyte growth factor leads to attenuations of tubular apoptosis and renal dysfunction in mouse ischemic kidneys. Am J Pathol 166: 1895-1905, 2005.

8. Ohnishi H, Mizuno S and Nakamura T: Inhibition of tubular cell proliferation by neutralizing endogenous HGF leads to renal hypoxia and bone marrow-derived cell engraftment in acute renal failure. Am J Physiol Renal Physiol 294: F326-F335, 2008.
9. Kato T, Funakoshi H, Kadoyama K, Noma S, Kanai M, Ohya-Shimada W, Mizuno S, Doe N, Taniguchi T and Nakamura T: Hepatocyte growth factor overexpression in the nervous system enhances learning and memory performance in mice. J Neurosci Res 90: 1743-1755, 2012.

10. MacGregor GG, Xu JZ, McNicholas CM, Giebisch G and Hebert SC: Partially active channels produced by PKA site mutation of the cloned renal $\mathrm{K}+$ channel, ROMK2 (kir1.2). Am J Physiol 275: F415-F422, 1998.

11. Yoo D, Kim BY, Campo C, Nance L, King A, Maouyo D and Welling PA: Cell surface expression of the ROMK (Kir 1.1) channel is regulated by the aldosterone-induced kinase, SGK-1, and protein kinase A. J Biol Chem 278: 23066-23075, 2003.

12. Lang $\mathrm{F}$ and Cohen $\mathrm{P}$ : Regulation and physiological roles of serum- and glucocorticoid-induced protein kinase isoforms. Sci STKE 2001: re17, 2001.

13. Náray-Fejes-Tóth A, Canessa C, Cleaveland ES, Aldrich G and Fejes-Tóth G: SGK is an aldosterone-induced kinase in the renal collecting duct. Effects on epithelial na+ channels. J Biol Chem 274: 16973-16978, 1999.

14. Shelly $\mathrm{C}$ and Herrera R: Activation of SGK1 by HGF, Rac1 and integrin-mediated cell adhesion in MDCK cells: PI-3K-dependent and -independent pathways. J Cell Sci 115: 1985-1993, 2002.

15. Bosch X, Poch E and Grau JM: Rhabdomyolysis and acute kidney injury. N Engl J Med 361: 62-72, 2009.

16. Ukai T: The Great Hanshin-Awaji Earthquake and the problems with emergency medical care. Ren Fail 19: 633-645, 1997.

17. Nagano T, Mori-Kudo I, Tsuchida A, Kawamura T, Taiji M and Noguchi H: Ameliorative effect of hepatocyte growth factor on glycerol-induced acute renal failure with acute tubular necrosis. Nephron 91: 730-738, 2002.

18. Ishibashi K, Sasaki S, Sakamoto H, Nakamura Y, Hata T, Nakamura T and Marumo F: Hepatocyte growth factor is a paracrine factor for renal epithelial cells: Stimulation of DNA synthesis and NA,K-ATPase activity. Biochem Biophys Res Commun 182: 960-965, 1992. 